\title{
Do microcracks decrease or increase fatigue resistance in cortical bone?
}

\author{
O.S. Sobelman ${ }^{\mathrm{a}}$, J.C. Gibeling ${ }^{\mathrm{b}}$, S.M. Stover ${ }^{\mathrm{c}}$, S.J. Hazelwood ${ }^{\mathrm{a}}$, O.C. Yeh ${ }^{\mathrm{a}}$, \\ D.R. Shelton ${ }^{\mathrm{b}}$, R.B. Martin ${ }^{\mathrm{a}, *}$ \\ ${ }^{a}$ Orthopaedic Research Laboratories, School of Medicine, University of California at Davis, Davis, CA 95616, USA \\ ${ }^{\mathrm{b}}$ Department of Chemical Engineering and Materials Science, College of Engineering, University of California at Davis, Davis, CA 95616, USA \\ ${ }^{\mathrm{c}}$ J.D. Wheat Veterinary Orthopedic Research Laboratory, School of Veterinary Medicine, University of California at Davis, Davis, CA 95616, USA
}

\begin{abstract}
Fatigue of cortical bone produces microcracks; it has been hypothesized that these cracks are analogous to those occurring in engineered composite materials and constitute a similar mechanism for fatigue resistance. However, the numbers of these linear microcracks increase substantially with age, suggesting that they contribute to increased fracture incidence among the elderly. To test these opposing hypotheses, we fatigued 20 beams of femoral cortical bone from elderly men and women in load-controlled four point bending having initial strain ranges of 3000 or 5000 microstrain. Loading was stopped at fracture or $10^{6}$ cycles, whichever occurred first, and microcrack density and length were measured in the loaded region and in a control region that was not loaded. We studied the dependence of fatigue life and induced microdamage on initial microdamage, cortical region, subject gender and age, and several other variables. When the effect of modulus variability was controlled, longer fatigue life was associated with higher rather than lower initial crack density, particularly in the medial cortex. The increase in crack density following fatigue loading was greater in specimens from older individuals and those initially having longer microcracks. Crack density increased as much in specimens fatigued short of the failure point as in those that fractured, and microcracks were, on average, shorter in specimens with greater numbers of resorption spaces, a measure of remodeling rate.
\end{abstract}

\section{Introduction}

Frost (1960) first identified short (ca. $80 \mu \mathrm{m})$, interlamellar cracks in human rib cross-sections as fatigue damage. Such "microcracks" are usually isolated in interstitial regions among more or less intact osteons (Norman and Wang, 1997; Schaffler et al., 1995). Microcracks occur naturally in vivo and subsequent to fatigue loading to physiologic strain levels (Burr et al., 1985; Mori and Burr, 1993; Schaffler et al., 1989, Schaffler et al., 1990). This paper is concerned with the mechanical significance of such isolated, physiologic microcracks, and draws a clear distinction between them and the much more extensive systems of longer cracks and diffuse damage associated with fracture or severe, non-physiologic loading (Boyce et al., 1998; Burr et al., 1998; Martin et al., 1996b; Zioupos and Currey, 1998).

Microcrack density (cracks $/ \mathrm{mm}^{2}$ of cross-section) increases with age in both cortical and trabecular bone (Fazzalari et al., 1998; Mori et al., 1997; Norman and Wang, 1997; Schaffler et al., 1995), suggesting that isolated microcracks contribute to bone weakness and fractures in the elderly. However, causal relationships between isolated microcracks and bone strength or fracture toughness have not been established. While loading bones to physiologic strain levels increases microcrack density, and severe loading results in much more extensive damage, so far there is little evidence that isolated microcracks significantly weaken cortical bone, or are associated with failure. Testing human cortical bone, Norman et al. (1998) found significant 
$(0.03<p<0.05)$ negative correlations between tensile fracture toughness and pre-existing microcrack densities and lengths in femurs, but not in tibias. Burr et al. (1998) found that flexural fatigue loading of canine femurs resulted in extensive networks of longer cracks and diffuse damage, but neither the density or lengths of microcracks was significantly increased. Similarly, O'Brien et al. (2003) found that microcrack growth and coalescence was not a significant factor in fatigue failure of bovine cortical bone. Consistent with fracture mechanics theory, these data suggest that bone fails by extensive propagation of a few cracks, not the proliferation and coalescence of microcracks.

Isolated, linear cracks associated with material interfaces have been studied in other load-bearing composite structures such as sedimentary geological formations and fiber-matrix materials (Fig. 1, upper left)(Reifsnider, 1991). Tensile loading of such materials initially produces cracks in planes perpendicular to the loading direction having dimensions limited by interactions with adjacent components of the composite (Bai et al., 2000; Chawla, 1998). However, as loading increases, cracks stop forming when the tensile stress between them is sufficiently diminished by their increasing proximity. It is thought that such cracks increase toughness by absorbing strain energy and redistributing stress (Akkus et al., 2000; Vashishth et al., 1997). Fracture is not

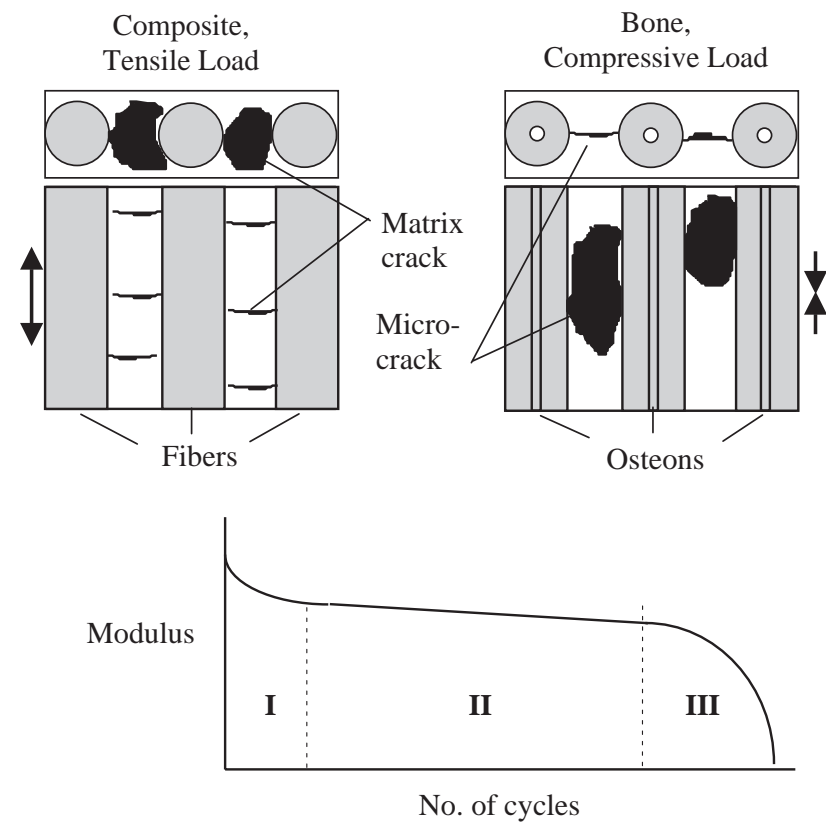

Fig. 1. At upper left is a schematic diagram depicting crack damage developed under a longitudinal tensile load in a fiber-matrix composite; cross-sectional view above, longitudinal view below. At upper right is a comparable diagram of a compressively loaded region of osteonal bone. Transverse cracks develop in the brittle matrix of the composite, and longitudinal cracks develop in the interstitial matrix of the bone. Shown below is the three-phase elastic modulus degradation curve characteristic of both the composite material and bone. approached until this preliminary form of damage has saturated and other forms of damage continue the failure process.

Reifsnider (1991) has related such preliminary, selflimiting damage to Phase I of the typical tripartite fatigue degradation curve for composite materials, in which elastic modulus declines as initial damage forms (Fig. 1, bottom). Subsequently, during Phase II of the fatigue life, modulus degradation proceeds more slowly as other forms of damage occur (e.g., fiber delamination and breaking), until the composite's integrity begins to fail rapidly in Phase III. Reifsnider refers to the situation in which transverse cracking has saturated as a "characteristic damage state," and regards this phenomenon as an important element in the design of materials to resist fatigue. Shum and Hutchinson (1990) proposed that the formation of self-limiting cracks in loaded structures serves to increase toughness by absorbing strain energy and redistributing stress.

In cortical bone, the lamellar structures associated with osteonal remodeling may create similar effects by facilitating the formation of microcracks having modest, self-limiting numbers and lengths (Martin and Burr, 1989; Mori and Burr, 1993; Vashishth et al., 2000). It is postulated that such controlled microscopic cracking toughens the material by redistributing stresses so that it is much more difficult to sustain the propagation of catastrophic cracks (Akkus et al., 2000; Vashishth et al., 1997).

There is, however, a basic difference between osteonal bone and the Reifsnider model. Long bones are characteristically loaded in bending rather than the simple tension depicted at upper left in Fig. 1. Furthermore, Boyce et al. (1998) observed that when cortical bone is subjected to bending, microcracks are characteristically found in the compressed region but not in the tension region. Therefore, to adapt the model to bone, it is appropriate to think in terms of longitudinal compression (Fig. 1, upper right). In this case, tensile stresses are due to a Poisson effect acting transversely rather than longitudinally. Also, most microcracks occur in the older interstitial matrix between intact osteons, consisting of isolated lamellar remnants of old osteons (Norman and Wang, 1997; Schaffler et al., 1995). Otherwise, the concept is similar: the formation of cracks having limited capacity to grow and multiply prolongs fatigue life via toughening and redistribution of stress. Furthermore, in bone's in vivo state, these toughening, linear microcracks activate remodeling (Bentolila et al., 1998; Mori and Burr, 1993), a mechanism for damage removal. Consequently, microcrack damage may be limited to values well short of saturation, allowing bone to remain in Phase I of the failure process indefinitely.

However, as noted above, studies have shown agerelated increases in linear microcrack density in human 
bone, and there is concern that this may contribute to the increased incidence of fractures in the elderly (Burr et al., 1997; Heaney, 1998). Here we ask, is greater microcrack density in cortical bone from elderly people evidence of fatigue weakness, or of fatigue resistance? In either case, as a predictor of subsequent fatigue life, how does microcrack density compare to age, gender, cortical region, and such tissue properties as mineralization and porosity? We hypothesize that modest numbers of isolated microcracks in cortical bone constitute a mechanism for fatigue resistance and such cracks will be more numerous in specimens subsequently exhibiting a longer fatigue life.

\section{Methods}

Twenty unpaired human femurs, evenly divided between male and female bones, were obtained from fresh cadavers, wrapped in saline soaked gauze, sealed in plastic bags, and stored at $-20^{\circ} \mathrm{C}$. Each sex was represented by 4 left and 6 right femurs. The subjects had no record of bone disease, and gross examination revealed no pathologic anatomical features. Age at death was $59-94 \mathrm{yr}$ (mean \pm standard deviation $=$ $75.1 \pm 8.6 \mathrm{yr}$ ). Keeping the bones wet, a beam was machined from the medial $(N=10)$ or lateral $(N=10)$ half of each femoral mid-diaphysis. The beams were uniformly $10 \mathrm{~mm}$ wide and $100 \mathrm{~mm}$ long; their thickness was $4 \mathrm{~mm}$ or as great as the cortical thickness would permit (mean $3.34 \pm 0.56 \mathrm{~mm}$ ). The fatigue loading protocol was similar to that of Griffin et al. (1997), producing symmetric four-point bending on an Instron 1321 testing machine (Instron Corporation, Canton MA) using an MTS TestStar II digital controller and Testware-SX software (MTS, Eden Prairie, MN). Fixturing included $9.53 \mathrm{~mm}$ diameter outer and inner stainless steel roller supports, symmetrically placed 64 and $32 \mathrm{~mm}$ apart, respectively (ASTM Standard D790M-82), and a 4L immersion tank containing calcium buffered saline (Gustafson et al., 1996) at $37^{\circ} \mathrm{C}$. Beams were fatigued with the periosteal surface in tension using a $2 \mathrm{~Hz}$, sinusoidally varying load measured by an MTS model 661.18C-02, $1200 \mathrm{~N}$ load cell. Center deflection was measured using a DC-E 125 LVDT (Schavitz Engineering, Pennsauken, NJ). Specimens were fatigued under a constant load range calculated to produce either $3000(n=7)$ or 5000 $(n=13)$ peak microstrain on the initial cycle (initial microstrain, $i \mu \varepsilon)$. The $3000 i \mu \varepsilon$ range approximated the upper physiologic limit (Rubin and Lanyon, 1985), above the $2500 \mathrm{i} \mu \varepsilon$ tensile damage threshold of Pattin et al. (1996). The $5000 \mathrm{i} \mu \varepsilon$ range ensured that some specimens would break within $10^{6}$ cycles, a practical limit.
Each specimen was thawed and equilibrated to $37^{\circ} \mathrm{C}$. Using beam theory, the initial tangent modulus was obtained from the mean slope of three load-deflection curves to $50 \mathrm{~N}$, well within the elastic range. The force necessary to obtain the desired maximum i $\mu \varepsilon$ was then calculated, and the specimen was loaded between $10 \mathrm{~N}$ and this force until complete fracture occurred (fatigue life $=N_{\mathrm{f}}$ cycles), or for $10^{6}$ cycles, whichever occurred first.

Following fatigue loading, unfractured beams were transected at their midpoint using a Leitz model 1600 diamond saw (Leica, Deerfield, IL) to produce a pseudo-fracture site. Both pieces of fractured and cut specimens were bulk-stained in basic fuchsin (Burr and Hooser, 1995), and six cross-sections ( $100 \pm 15 \mu \mathrm{m}$ thick) were cut from the loaded portion of each beam, three from each side of the fracture site and $2-3 \mathrm{~mm}$ from the fracture surface, using the Leitz saw (Fig. 2). We thereby obtained sections representative of the material in the loaded region excluding the macrocracks immediately adjacent to the fracture surfaces. Three similar sections were cut from each load-free region beyond the outer supports. All 12 sections were mounted on glass slides for analysis.

Two $5 \times 10 \times 4 \mathrm{~mm}$ specimens were cut from each beam, one just beyond the outer support and one just within the inner support at one end (Fig. 2). The volume (V) of each was calculated from its dimensions, measured with a micrometer $( \pm 0.01 \mathrm{~mm})$. Using a Mettler Toledo AB204 balance ( $\pm 0.1 \mathrm{mg}$, Switzerland), each specimen was weighed after removal from water and blotting with a tissue (wet mass, $M_{\mathrm{W}}$ ), after drying for $24 \mathrm{~h}$ at $100^{\circ} \mathrm{C}$ (dry mass, $M_{\mathrm{D}}$ ), and after ashing for $48 \mathrm{~h}$ in a muffle furnace at $800^{\circ} \mathrm{C}$ (ash mass, $M_{\mathrm{A}}$ ). Wet and dry apparent density, and percent ash weight were calculated as $D_{\mathrm{W}}=M_{\mathrm{W}} / V, D_{\mathrm{D}}=M_{\mathrm{D}} / V$, and $\%$ Ash $=100 M_{\mathrm{A}} / M_{\mathrm{D}}$, respectively.

Using an Olympus Model BH-2 microscope (Olympus, Lake Success, NY) histomorphometric measurements were made at $125 \mathrm{x}$ over the entire area of all 12

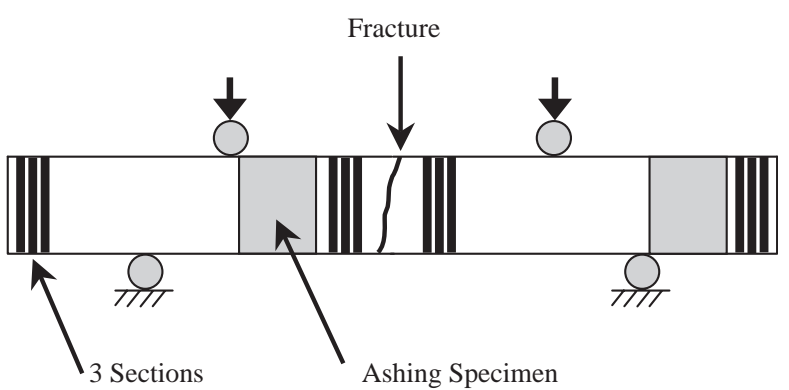

Fig. 2. Side view of beam showing approximate locations of the analysis specimens. Twelve histologic sections are represented by black bands, and two specimens for density and \%Ash measurements are shown as gray boxes. Circles represent the four-point bending roller supports. (Not to scale.) 
sections from each beam. Porosity was obtained using a Merz grid, counting the fraction of points falling within Haversian canals, resorption spaces, and Volkmann's canals, but not smaller voids (e.g., osteocyte lacunae). Active remodeling was quantified as the population density of basic multicellular unit (BMU) resorption spaces (Rs.Ar, $\mathrm{mm}^{-2}$ ). Using the method of Martin et al. (1996a), a measure of longitudinal collagen fiber orientation known as the "longitudinal structure index" (LSI) was obtained for each section using circularly polarizing filters. (Higher values of LSI correspond to greater proportions of longitudinally oriented collagen fibers.)

Microcrack density (Cr.Dn, $\mathrm{mm}^{-2}$ ) and microcrack length $(\mathrm{Cr}$.Le, $\mathrm{mm}$, measured using an eyepiece graticule) were measured using the criteria of Burr and Stafford (Burr and Stafford, 1990). Crack surface density (total crack length per unit area, Cr.S.Dn, $\mathrm{mm}^{-1}$ ) was calculated as the product of Cr.Dn. and mean Cr.Le. Microdamage assessment did not include diffuse microdamage (Schaffler et al., 1994), or components of the extensive systems of cracks associated with failure. Rather, measurements were restricted to the archetypical, isolated, linear cracks described by Frost (1960) and others (Burr and Hooser, 1995; Burr and Stafford, 1990; Norman and Wang, 1997; Schaffler et al., 1995). Microdamage measurements from the load-free regions at the ends of each beam were assumed to represent initial, pre-fatigue values, to be compared with post-loading data from the central portion of the beam.

Statview for Windows Version 5.01 (SAS Institute, Inc., Cary, NC) was used for statistical analyses with $p<0.05$ the criterion for significance. Using analysis of variance methods with age as a covariate, we tested all variables for the effects of gender, side (left or right), and region (lateral or medial cortex). We examined the effect of fatigue loading using paired $t$-tests to compare initial and post-loading values, and we tested the dependence of the mechanical variables on the histomorphometric and compositional variables using regression analyses. Control (initial) and post-loading microdamage variables are indicated by $i$ and $p$ prefixes, respectively: iCr.Dn and pCr.Dn, etc.

\section{Results}

While iCr.Dn, iCr.Le, and iCr.S.Dn exhibited Poisson-like distributions, pCr.Dn, pCr.Le, and pCr.S.Dn in the loaded sections were more normally distributed. Rs.Ar also had a Poisson-like distribution. We therefore used nonparametric methods or a square root transformation in statistical tests involving Poisson-like variables. (N.B.: Square root transformed variables are indicated by a superscript T, e.g. iCr.Dn ${ }^{\mathrm{T}}$.) Except for microdamage, the variables measured within the loaded
Table 1

Mean and standard deviation (SD) values for male and female specimens

\begin{tabular}{llll}
\hline Variables & $\begin{array}{l}\text { Males } \\
\text { Mean } \pm \text { SD }\end{array}$ & $\begin{array}{l}\text { Females } \\
\text { Mean } \pm \text { SD }\end{array}$ & $p$ \\
\hline Age $(\mathrm{yr})$ & $76.4 \pm 8.8$ & $73.7 \pm 8.5$ & 0.50 \\
Modulus, GPa & $17.60 \pm 2.04$ & $16.57 \pm 2.459$ & 0.32 \\
Log $N_{\mathrm{f}}$ & $4.813 \pm 1.151$ & $5.028 \pm 0.650$ & 0.63 \\
iCr.Dn $\left(\mathrm{mm}^{-2}\right)$ & $0.155 \pm 0.122$ & $0.255 \pm 0.307$ & 0.35 \\
pCr.Dn $\left(\mathrm{mm}^{-2}\right)$ & $0.283 \pm 0.227$ & $0.308 \pm 0.227$ & 0.81 \\
iCr.Le $\left(\mathrm{mm}^{-2}\right.$ & $0.0798 \pm 0.0216$ & $0.0791 \pm 0.0179$ & 0.94 \\
pCr.Le $\left(\mathrm{mm}^{2}\right)$ & $0.0962 \pm 0.0309$ & $0.0945 \pm 0.0206$ & 0.89 \\
iCr.S.Dn $\left(\mathrm{mm}^{-1}\right)$ & $0.0147 \pm 0.0114$ & $0.0240 \pm 0.0306$ & 0.38 \\
pCr.S.Dn $\left(\mathrm{mm}^{-1}\right)$ & $0.0272 \pm 0.0207$ & $0.0307 \pm 0.0227$ & 0.73 \\
Porosity $(\%)$ & $9.59 \pm 2.13$ & $11.81 \pm 5.02$ & 0.21 \\
Rs.Ar $\left(\mathrm{mm}^{-2}\right)$ & $0.118 \pm 0.086$ & $0.096 \pm 0.077$ & 0.55 \\
LSI & $6.06 \pm 0.44$ & $6.31 \pm 0.49$ & 0.24 \\
Wet Den $\left(\mathrm{gm} \mathrm{cm}^{-3}\right)$ & $1.97 \pm 0.09$ & $1.92 \pm 0.13$ & 0.43 \\
Dry Den $\left(\mathrm{gm} \mathrm{cm}^{-3}\right)$ & $1.71 \pm 0.080$ & $1.66 \pm 0.13$ & 0.39 \\
\% Ash & $66.87 \pm 0.66$ & $66.64 \pm 1.17$ & 0.58 \\
\hline
\end{tabular}

$p$-values show no gender differences. Note that the mean and SD of $\log N_{\mathrm{f}}$ represent only those specimens loaded at $5000 \mathrm{i} \mu \varepsilon$, and data in the bottom six rows represent combined data from portions of the beam that were, and were not, loaded. $N=10$ males, 10 females for all variables except $\log N_{\mathrm{f}}$ : seven males, six females.

and load-free portions of each beam were not significantly different, and were averaged together for subsequent analyses. Table 1 shows basic statistics by gender for the principal variables. With a single exception (regional difference in \%Ash, see below), there were no gender, side, or regional differences when these variables were considered independently of one another.

Of the seven specimens fatigued at $3000 \mathrm{i} \mu \varepsilon$ for up to $10^{6}$ cycles, only one failed (at 319,553 cycles). The fatigue lives of the 13 specimens loaded at $5000 \mathrm{i} \mu \varepsilon$ ranged from 433 to 925,766 cycles. When $\log N_{\mathrm{f}}$ for the $5000 \mathrm{i} \mu \varepsilon$ specimens was regressed against the $\log$ of initial strain $(\varepsilon$, microstrain) along with similar data for human femoral bone tested in our laboratory at 6000 , 6500 , and $7500 \mathrm{i} \mu \varepsilon$ (Griffin et al., 1997), the result was

$N_{\mathrm{f}}=3.68 \times 10^{49} \varepsilon^{-12.1}$

$\left(r^{2}=0.556, p<0.0001\right)$. This equation predicts $N_{\mathrm{f}}$ for $3000 \mathrm{i} \mu \varepsilon$ to be $31.1 \times 10^{6}$ cycles, consistent with the failure of only one $3000 \mathrm{i} \mu \varepsilon$ specimen at $<10^{6}$ cycles.

When $\log N_{\mathrm{f}}$ was regressed individually against the other variables in Table 1, only elastic modulus was statistically significant, with less stiff bones having longer fatigue lives $\left(y=8.70-0.221 x, \quad r^{2}=0.350\right.$, $p=0.0332$ ). Elastic modulus in turn was significantly correlated only with wet density $(y=-3.42+10.5 x$, $\left.r^{2}=0.271, \quad p=0.0186\right) \quad$ and dry density $\left(y=-3.37+12.1 x, r^{2}=0.329, p=0.0081\right)$.

Stepwise multiple linear regression revealed additional relationships between the variables. When 
Table 2

Multiple regression analyses for $\log N_{\mathrm{f}}$ vs. modulus and (upper panel) cortical region or (lower panel) initial crack density

\begin{tabular}{|c|c|c|}
\hline \multirow[t]{2}{*}{$\log N_{\mathrm{f}}$ vs. } & \multicolumn{2}{|c|}{$r^{2}=0.579, p=0.0133$} \\
\hline & Coefficient & $p$ \\
\hline Modulus & -0.271 & 0.0067 \\
\hline Cortical region $^{\mathrm{a}}$ & -0.883 & 0.0420 \\
\hline Intercept & 10.848 & $<0.0001$ \\
\hline \multirow[t]{2}{*}{$\log N_{\mathrm{f}}$ vs. } & \multicolumn{2}{|c|}{$r^{2}=0.575, p=0.0139$} \\
\hline & Coefficient & $p$ \\
\hline Modulus & -0.255 & 0.0088 \\
\hline iCr. $\mathrm{Dn}^{\mathrm{T}}$ & 1.868 & 0.0443 \\
\hline Intercept & 8.598 & $<0.0001$ \\
\hline
\end{tabular}

${ }^{\mathrm{a}} 0$ - medial, 1-lateral.

the influence of elastic modulus, $E$, on $\log N_{\mathrm{f}}$ was controlled by multiple regression analysis, specimens from the medial region, and those having a larger value of $\mathrm{iCr} . \mathrm{Dn}^{\mathrm{T}}$, were seen to have a significantly longer fatigue life (Table 2). Rearranging the multiple regression equation represented by the lower panel of Table 2, one has

$\log N_{\mathrm{f}}+0.255 E=8.598+1.868$ iCr.Dn ${ }^{\mathrm{T}}$.

The left side of this equation may be considered a measure of fatigue life adjusted for modulus variation. When this was computed for each specimen and plotted against $\mathrm{iCr} . \mathrm{Dn}^{\mathrm{T}}$, the influence of initial microcrack density on modulus-adjusted fatigue life was seen to be positive (Fig. 3). Overall, this correlation was significant but relatively weak $\left(r^{2}=0.337, p=0.0377\right)$. However, it was strong for specimens from the medial region $\left(r^{2}=0.882, p=0.0017\right)$. This regional distinction was supported by two other findings. First, $t$-tests showed only one microstructural difference between the medial and lateral regions: \%Ash was significantly greater in the medial region $(67.172 \pm 0.653$ vs. $66.338 \pm 1.014$, $p=0.0423$ ). Over all specimens, $\mathrm{iCr} \cdot \mathrm{Dn}^{\mathrm{T}}$ increased in proportion to $\%$ Ash $\left(y=-10.71+0.166 x, r^{2}=0.458\right.$, $p=0.0010)$. Thus, there were consistent associations between the medial cortex, greater $\% \mathrm{Ash}, \mathrm{iCr} . \mathrm{Dn}^{\mathrm{T}}$, and $\log N_{\mathrm{f}}$.

$t$-Tests showed no differences in pCr.Dn, pCr.Le, or pCr.S.Dn for specimens fatigued at 3000 and $5000 \mathrm{i} \mu \varepsilon$. We therefore pooled the specimens and using Wilcoxon signed ranks tests we found that Cr.Dn, Cr.Le, and Cr.S.Dn were all significantly greater in the loaded region than in the control region (Table 3).

Stepwise linear regressions were performed to determine how other variables affected the microdamage produced by fatigue loading, irrespective of failure (i.e.,

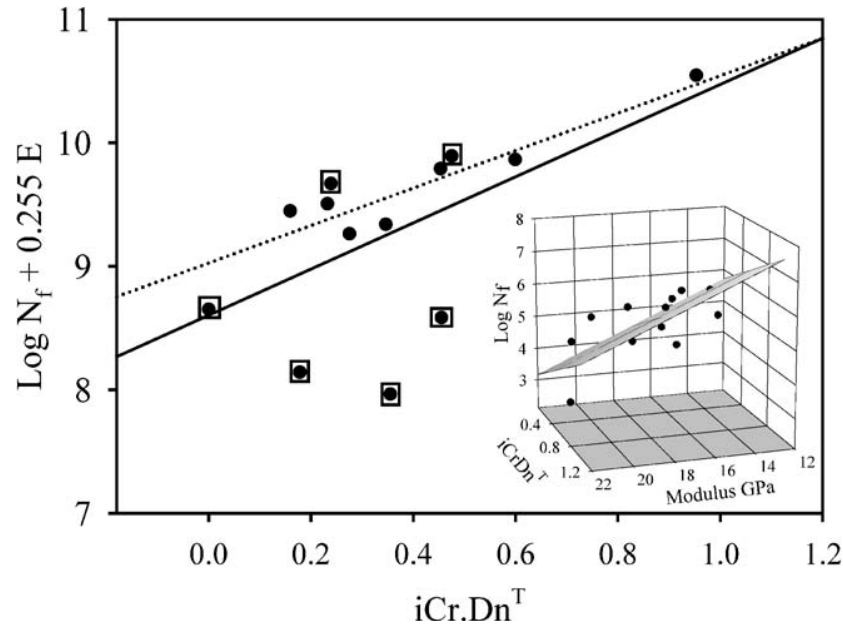

Fig. 3. Graphs showing relationships between $\log N_{\mathrm{f}}$, elastic modulus, and square root transformed initial Cr.Dn (iCr.Dn ${ }^{\mathrm{T}}$ ) for specimens fatigued at $5000 \mathrm{i} \mu \varepsilon$. Main graph shows modulus-adjusted $\log N_{\mathrm{f}}$ vs. iCr.Dn ${ }^{\mathrm{T}}$ (solid regression line, $y=8.111+1.811 x, \quad r^{2}=0.337$, $p=0.0377)$. Boxed points are from the lateral region; others are from the medial region, in which the effect of $\mathrm{iCr}$.Dn on modulus-adjusted $\log N_{\mathrm{f}}$ is much stronger (dotted line, $y=8.512+1.478 x, r^{2}=0.882$, $p=0.0017)$. Inset shows data points and plane of regression equation (Table 2, lower panel)

Table 3

Means and standard deviations for microdamage measures in control and loaded regions, and corresponding Wilcoxon signed rank test $p$ values. Pooled data for specimens tested at 3000 and $5000 \mathrm{i} \mu \varepsilon$

\begin{tabular}{llll}
\hline & Control & Loaded & $p$ \\
\hline Cr.Dn $\left(\mathrm{mm}^{-2}\right)$ & $0.205 \pm 0.233$ & $0.295 \pm 0.221$ & 0.0251 \\
Cr.Le $(\mathrm{mm})$ & $0.075 \pm 0.026$ & $0.095 \pm 0.026$ & 0.0206 \\
Cr.S.Dn $\left(\mathrm{mm}^{-1}\right)$ & $0.019 \pm 0.023$ & $0.029 \pm 0.021$ & 0.0111 \\
\hline
\end{tabular}

considering all 20 specimens). More than half the variability in $\mathrm{pCr} . \mathrm{Dn}^{\mathrm{T}}$ was related to the variability in iCr.Dn ${ }^{\mathrm{T}}\left(y=0.250+0.662 x, r^{2}=0.631, p<0.0001\right)$. Multiple linear regression showed that $\mathrm{pCr} \cdot \mathrm{Dn}^{\mathrm{T}}$ was also positively correlated with age (Table 4, upper panel). By moving the $\mathrm{iCr} . \mathrm{Dn}^{\mathrm{T}}$ term to the left side, this regression equation can be written as

pCr.Dn ${ }^{\mathrm{T}}-0.624 \mathrm{iCr} \cdot \mathrm{Dn}^{\mathrm{T}}=0.00638 \mathrm{Age}-0.214$.

The left side of this equation may be considered a measure of post-load Cr.Dn that is adjusted for variations in the initial crack density. This "adjusted post-load Cr.Dn" increased significantly with age (Fig. 4). However, all of the specimens that did not fracture lie above the regression line, supporting the hypothesis that abundant fatigue-induced microcracking is not associated with fracture. The analysis was therefore repeated using fatigue fracture $(1=$ yes, $0=$ no $)$ as a covariate along with age. This showed the adjusted post-load Cr.Dn to be negatively correlated 
Table 4

Multiple regression results showing variables that were significantly correlated with pCr.Dn ${ }^{\mathrm{T}}, \Delta \mathrm{Cr} . \mathrm{Dn}^{\mathrm{T}}$, and $\mathrm{iCr} \cdot \mathrm{Le}^{\mathrm{T}}$

\begin{tabular}{llr}
\hline$p C r . D n^{T} v s$. & $r^{2}=0.710, p<0.0001$ & \multicolumn{1}{c}{} \\
\cline { 2 - 3 } & Coefficient & $<0.0001$ \\
\hline iCr.Dn $^{\mathrm{T}}$ & 0.624 & 0.0458 \\
Age & 0.00638 & 0.3458
\end{tabular}

$\triangle C r \cdot D n^{T}$ vs. $\quad r^{2}=0.732, p=0.0006$

\begin{tabular}{lrr}
\cline { 2 - 3 } & Coefficient & \multicolumn{1}{c}{$p$} \\
\hline iCr.Dn $^{\mathrm{T}}$ & -0.794 & $<0.0001$ \\
Age & 0.009 & 0.0018 \\
iCr.Le & 2.515 & 0.0079 \\
LSI & 0.150 & 0.0086 \\
Intercept & -1.906 & 0.0086
\end{tabular}

\begin{tabular}{llr}
\hline$i C r . L e^{T}$ vs. & $r^{2}=0.647, p=0.0002$ & \\
\cline { 2 - 3 } & Coefficient & \multicolumn{1}{c}{$p$} \\
\hline Rs.Ar $^{\mathrm{T}}$ & -0.144 & 0.0054 \\
iCr.Dn $^{\mathrm{T}}$ & 0.078 & 0.0102 \\
Intercept & 0.291 & $<0.0001$ \\
\hline
\end{tabular}

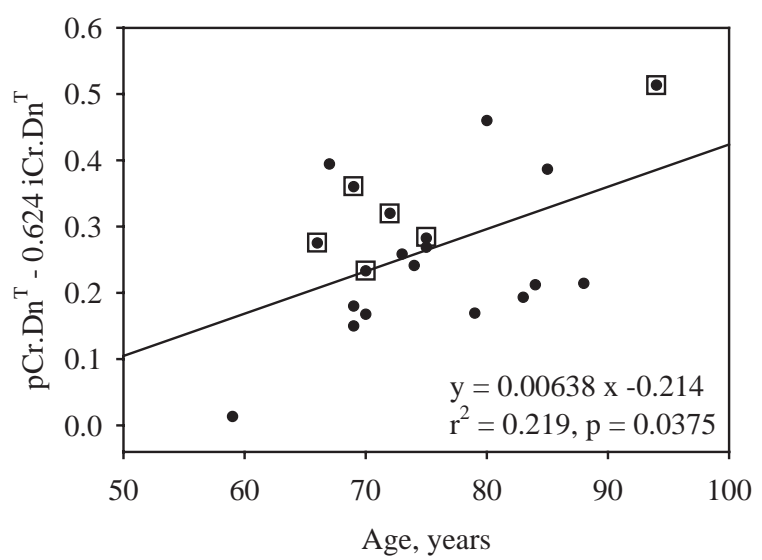

Fig. 4. Graph of $\mathrm{pCr} \cdot \mathrm{Dn}^{\mathrm{T}}$ adjusted for initial values vs. age (see Eq. (3)). Boxed points are specimens that did not fracture.

with fatigue fracture $(p=0.0478)$, and including this in the regression improved the significance of age as a predictor of post-load Cr.Dn to $p=0.0197$.

Multiple regression analysis showed that the postload change in crack density $\left(\Delta \mathrm{Cr} \cdot \mathrm{Dn}^{\mathrm{T}}=\right.$ $\left.p C r \cdot D n^{\mathrm{T}}-\mathrm{iCr} \cdot \mathrm{Dn}^{\mathrm{T}}\right)$ was negatively correlated with iCr.Dn ${ }^{\mathrm{T}}$ (as would be expected), and positively correlated with age, iCr.Le ${ }^{\mathrm{T}}$, and LSI (Table 4 , middle). Finally, iCr.Le ${ }^{\mathrm{T}}$ was correlated positively with iCr.Dn ${ }^{\mathrm{T}}$ and negatively with $\mathrm{Rs}^{-A^{\mathrm{T}}}{ }^{\mathrm{T}}$ (Table 4, bottom). Rs.Ar ${ }^{\mathrm{T}}$ alone accounted for nearly half the variability in iCr.Le ${ }^{\mathrm{T}}$ (Fig. 5).

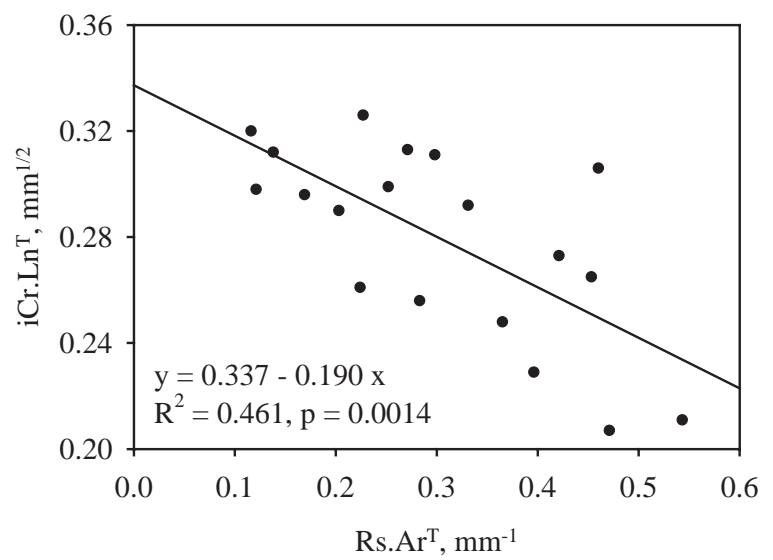

Fig. 5. Graph of iCr.Le ${ }^{T}$ vs. $\operatorname{Rs} \cdot \mathrm{Ar}^{\mathrm{T}}$ for all 20 specimens. The distribution of points about the regression line was not biased by age, sex, cortical region, or side.

\section{Discussion}

We found that when the effect of modulus variability was controlled, longer fatigue life was associated with greater rather than lower iCr.Dn, particularly in the medial cortex. Adjusting for variation in iCr.Dn, fatigue loading increased $\mathrm{Cr}$.Dn more in specimens from older individuals and those initially having longer cracks. Cr.Dn increased as much in specimens fatigued short of the failure point as in those that fractured, and iCr.Le was negatively correlated with remodeling activity (Rs.Ar).

This study was subject to several important limitations, the most important being the relatively small sample size. As is evident from Table 1, our sample size did not provide the statistical power to detect gender differences in the measured variables, and hampered other aspects of the analysis. We studied only older individuals because of their expected greater variability in $\mathrm{iCr} . \mathrm{Dn}$, and to test whether the increased Cr.Dn commonly observed in this population implies diminished resistance to fatigue failure. However, this choice meant that we also did not have the power needed to show the effects of age on variables such as a fatigueinduced increase in crack density without simultaneously accounting for other variables, such as SRT iCr.Dn and LSI. Clearly, future work should expand the numbers presented here.

We limited our testing to load control to defined initial strains because adult animals generally maintain approximately constant body weight during most of their lives, suggesting their bones function under something like "load control." Another limitation was the fact that we fatigued our specimens at superphysiologic strains because reproducing physiologic strains would have required impractically long fatigue tests. We therefore aimed to uniformly exceed physiologic strain 
levels. We limited our analysis to isolated microcracks because they correspond to the hypothetical mechanism for controlling fatigue damage described in the Introduction. In another flexural fatigue study of human femoral cortical bone, Boyce et al. (1998) observed that such microcracks were found primarily in the compressed region, with finer, "whispy" cracks near the neutral axis and diffuse damage zones in the tensile region. It is noteworthy that both the microcracks on the compressed side and the diffuse staining on the tensile side were preferentially located in interstitial zones. If the model suggested in Fig. 1 is valid, one would expect lamellae in the interstitial zones to experience lateral (Poisson) compressive forces on the tension side of the beam. Perhaps such lamellar compression produces damage to the mineralizedcollagen structure, and/or the osteocyte-canalicular network, that promotes diffusive stain infiltration.

Cr.Dn values reported here are comparable to human femoral values reported elsewhere (Boyce et al., 1998; Norman and Wang, 1997; Schaffler et al., 1995). While other authors' Cr.Dn data exhibited age-related increases (Norman and Wang, 1997; Schaffler et al., 1995), our iCr.Dn values did not, probably because all our specimens were from subjects aged $\geqslant 59 \mathrm{yr}$. Beyond age 60 there is a substantial increase in the variability as well as the mean of microdamage values in trabecular as well as cortical bone (Fazzalari et al., 1998; Mori et al., 1997; Norman and Wang, 1997; Schaffler et al., 1995). This suggests that microdamage increases much more in some individuals than in others, and the implications of this with regard to the underlying reasons and mechanical implications are unclear but intriguing.

We have consistently found that fatigue life is more dependent on initial elastic modulus than measures of tissue structure or composition. We attribute this to testing under load control to a defined initial strain (Boyce et al., 1998; Gibson et al., 1995; Pattin et al., 1996). This results in stiffer specimens being loaded to greater stresses than more compliant specimens, and $\log N_{\mathrm{f}}$ being inversely proportional to elastic modulus.

The analysis suggested that when the stiffness influence is controlled, fatigue life is greater in the medial than the lateral region. Portigliatti-Barbos et al. (1987) found that the femoral medial cortex was more mineralized than the lateral cortex, consistent with our observation concerning $\%$ Ash. We also found a positive association between fatigue life and iCr.Dn. These observations suggest that increased flexural fatigue life is favored rather than compromised by the initial presence of microcracks, which are in turn associated with bone that is slightly (but significantly) more mineralized.

Our goal was not to study damage clearly associated with fracture, but to focus on the relationship of fatigue life to isolated microcracks and other aspects of the bone tissue. We found that while fatigue loading increased Cr.Dn, the increase was age-dependent but independent of whether the initial strain was 5000 or $3000 \mathrm{i} \mu \varepsilon$, and not directly associated with fatigue failure. This suggests that fatigue-induced increases in microcrack density begin relatively early in the fatigue process and may have a self-limiting aspect. Others have shown that relatively modest, physiologic levels of fatigue loading result in small but significant increases in microcrack numbers (Burr et al., 1985; Mori and Burr, 1993; Schaffler et al., 1989, Schaffler et al., 1990). More severe fatigue loading, leading to substantial reductions in modulus and/or fracture, is associated with extensive diffuse damage and much longer cracks (Boyce et al., 1998; Burr et al., 1998; Martin et al., 1996b).

"Microcrack toughening" in which small cracks, with limited capacity to extend, reduce the strain energy release rate of larger cracks (Shum and Hutchinson, 1990), may result from microcracks in the interstitial regions of osteonal bone, where their capacity to propagate is limited by adjacent cement lines. Our results suggest that isolated microcracks may contribute to fatigue resistance. Clearly, however, there should be limits to this phenomenon. If our interpretation of these results in the context of the Reifsnider theory is correct, Cr.Dn should saturate and lead to other, more debilitating forms of damage, such as osteonal debonding and breakage. Also, a fundamental tenet of fracture mechanics is that short cracks remain stable, while those exceeding a critical length grow. Thus, control of microcracks' length appears essential to prevent more severe damage and fracture. There appear to be two elements to such control: the tendency of microcracks to form in interstitial regions where propagation is limited by cement lines of adjacent osteons, and the partial removal of cracks by BMUs.

While BMUs originate near microcracks, there is no evidence that they are guided to remove them entirely, and this is supported by our observation that Rs.Ar correlated negatively with iCr.Le (Fig. 5) but not iCr.Dn. In concert with this, Mashiba et al. (2000) found that reduction of canine cortical bone remodeling using risedronate or alendronate significantly increased Cr.Le in the rib, but only alendronate significantly increased Cr.Dn.

Controlling for iCr.Dn, we found that pCr.Dn following defined amounts of fatigue loading was greater in specimens from more aged individuals (Fig. 4). This suggests that a given amount of loading produces more microcracks in older individuals, and implies an age-related increase in susceptibility to microcracking. It is interesting to consider this observation in the context of the substantial but highly variable increase in Cr.Dn observed in people over age 60 (Norman and Wang, 1997; Schaffler et al., 1995), and to ask: Are those who exhibit greater age-related increases 
in Cr.Dn more susceptible to fracture, or are they are simply those who loaded their bones more during old age?

Clearly, the data presented here are not conclusive and raise many additional questions about bone's fatigue resistance mechanisms. However, these data seem to suggest that isolated microcracks are not harbingers of fatigue failure. Instead, short microcracks, typically confined to interstitial regions bound by osteonal cement lines and controlled by remodeling, may be agents of stress redistribution and fatigue life enhancement.

\section{Acknowledgements}

Supported by NIH Grant AR41644 and the Doris Linn Chair endowment (held by RBM).

\section{References}

Akkus, O., Jepsen, K.J., Rimnac, C.M., 2000. Microstructural aspects of the fracture process in human cortical bone. Journal of Materials Science 35, 1-10.

Bai, T., Pollard, D.D., Gao, H., 2000. Explanation for fracture spacing in layered materials. Nature 403, 753-756.

Bentolila, V., Boyce, T.M., Fyhrie, D.P., Drumb, R., Skerry, T.M., Schaffler, M.B., 1998. Intracortical remodeling in adult rat long bones after fatigue loading. Bone 23, 275-281.

Boyce, T.M., Fyhrie, D.P., Glotkowski, M.C., Radin, E.L., Schaffler, M.B., 1998. Damage type and strain mode associations in human compact bone bending fatigue. Journal of Orthopaedic Research $16,322-329$.

Burr, D.B., Forwood, M.R., Fyhrie, D.P., Martin, R.B., Schaffler, M.B., Turner, C.H., 1997. Bone microdamage and skeletal fragility in osteoporotic and stress fractures. J Bone and Mineral Research $12,6-15$.

Burr, D.B., Hooser, M., 1995. Alterations to the en bloc basic fuchsin staining protocol for the demonstration of microdamage produced in vivo. Bone 17, 431-433.

Burr, D.B., Martin, R.B., Schaffler, M.B., Radin, E.L., 1985. Bone remodeling in response to in vivo fatigue microdamage. Journal of Biomechanics 18, 189-200.

Burr, D.B., Stafford, T., 1990. Validity of the bulk-staining technique to separate artifactual from in vivo bone microdamage. Clinical Orthopaedics and Related Research 260, 305-308.

Burr, D.B., Turner, C.H., Naick, P., Forwood, M.R., Ambrosius, W., Hasan, M.S., Pidiparti, R., 1998. Does microdamage accumulation affect the mechanical properties of bone? Journal of Biomechanics 31, 337-345.

Chawla, K.K., 1998. Composite Materials Science and Engineering. Springer, New York.

Fazzalari, N.L., Forwood, M.R., Smith, K., Manthey, B.A., Herreen, P., 1998. Assessment of cancellous bone quality in severe osteoarthrosis: bone mineral density, mechanics, and microdamage. Bone 22, 381-388.

Frost, H.M., 1960. Presence of microscopic cracks in vivo in bone. Henry Ford Hospital Medical Bulletin 8, 25-35.

Gibson, V.A., Stover, S.M., Martin, R.B., Gibeling, J.C., Gustafson, M.B., Griffin, L.V., 1995. Fatigue behavior of the equine third metacarpus: mechanical property analysis. Journal of Orthopaedic Research 13, 861-868.

Griffin, L.V., Gibeling, J.C., Martin, R.B., Gibson, V.A., Stover, S.M., 1997. A model of flexural fatigue damage accumulation for cortical bone. Journal of Orthopaedic Research 15, 607-614.

Gustafson, M.B., Martin, R.B., Gibson, V., Storms, D.H., Stover, S.M., Gibeling, J., Griffin, L., 1996. Calcium buffering is required to maintain bone stiffness in saline solution (technical note) Journal of Biomechanics 29, 1191-1194.

Heaney, R.P., 1998. Pathophysiology of osteoporosis. Endocrinology and Metabolism Clinics of North America 27, 255-265.

Martin, R.B., Burr, D.B., 1989. The Structure, Function, and Adaptation of Compact Bone. Raven Press, New York.

Martin, R.B., Mathews, P.V., Lau, S.T., Gibson, V.A., Stover, S.M., 1996a. Collagen fiber organization is related to mechanical properties and remodeling in equine bone. A comparison of two methods. Journal of Biomechanics 29, 1515-1521.

Martin, R.B., Stover, S.M., Gibson, V.A., Gibeling, J.C., Griffin, L.V., 1996b. In vitro fatigue behavior of the equine third metacarpus: remodeling and microcrack damage analysis. Journal of Orthopaedic Research 14, 794-801.

Mashiba, T., Hirano, T., Turner, C.H., Forwood, M.R., Johnston, C.C., Burr, D.B., 2000. Suppressed bone turnover by bisphosphonates increases microdamage accumulation and reduces some biomechanical properties in dog rib. Journal of Bone and Mineral Research 15, 613-620.

Mori, S., Burr, D.B., 1993. Increased intracortical remodeling following fatigue damage. Bone 14, 103-109.

Mori, S., Harruff, R., Ambrosius, W., Burr, D.B., 1997. Trabecular bone volume and microdamage accumulation in the femoral heads of women with and without femoral neck fractures. Bone 21, 521-528.

Norman, T.L., Wang, Z., 1997. Microdamage of human cortical bone: incidence and morphology in long bones. Bone 20, 375-379.

Norman, T.L., Yeni, Y.N., Brown, C.U., Wang, Z., 1998. Influence of microdamage on fracture toughness of the human femur and tibia. Bone 23, 303-306.

O'Brien, F.J., Taylor, D., Lee, T.C., 2003. Microcrack accumulation at different intervals during fatigue testing of compact bone. Journal of Biomechanics 36, 973-980.

Pattin, C.A., Caler, W.E., Carter, D.R., 1996. Cyclic mechanical property degradation during fatigue loading of cortical bone. Journal of Biomechanics 29, 69-80.

Portigliatti-Barbos, M., Carando, S., Ascenzi, A., Boyde, A., Improta, S., 1987. A biomechanical analysis at lamellar level of femoral shafts deformed in bending. In: Bergmann, G., Kobel, R., Rohlmann, A. (Eds.), Biomechanics: Basic and Applied Research. Martinus Nijhoff Publishers, Dordrecht, pp. 231-236.

Reifsnider, K.L., 1991. Damage and damage mechanics. In: Reifsnider, K.L. (Ed.), Fatigue of Composite Materials. Amsterdam, Elsevier, pp. 11-77.

Rubin, C.T., Lanyon, L.E., 1985. Regulation of bone mass by mechanical strain magnitude. Calcified Tissue International 37, 411-417.

Schaffler, M.B., Choi, K., Milgrom, C., 1995. Aging and matrix microdamage accumulation in human compact bone. Bone 17 , 521-525.

Schaffler, M.B., Pitchford, W.C., Choi, K., Riddle, J.M., 1994 Examination of compact bone microdamage using back-scattered electron microscopy. Bone 15, 488-493.

Schaffler, M.B., Radin, E.L., Burr, D.B., 1989. Mechanical and morphological effects of strain rate on fatigue of compact bone. Bone 10, 207-214.

Schaffler, M.B., Radin, E.L., Burr, D.B., 1990. Long-term fatigue behavior of compact bone at low strain magnitude and rate. Bone $11,321-326$. 
Shum, D.K.M., Hutchinson, J.W., 1990. On toughening by microcracks. Mechanics of Materials 9, 83-91.

Vashishth, D., Behiri, J.C., Bonfield, W., 1997. Crack growth resistance in cortical bone: concept of microcrack toughening. Journal of Biomechanics 30, 763-769.
Vashishth, D., Tanner, K.E., Bonfield, W., 2000. Contribution, development and morphology of microcracking in cortical bone during crack propagation. Journal of Biomechanics 33, 1169-1174.

Zioupos, P., Currey, J.D., 1998. Changes in the stiffness, strength, and toughness of human cortical bone with age. Bone 22, 57-66. 